\title{
Overexpression of interleukin-35 associates with hepatocellular carcinoma aggressiveness and recurrence after curative resection
}

\author{
Yi-Peng $\mathrm{Fu}^{1,4}$, Yong $\mathrm{Yi}^{1,4}$, Xiao-Yan Cai ${ }^{1,4}$, Jian Sun ${ }^{1}$, Xiao-Chun $\mathrm{Ni}^{1}$, Hong-Wei He ${ }^{1}$, Jia-Xing Wang ${ }^{1}$, \\ Zhu-Feng Lu' ${ }^{1}$, Jin-Long Huang ${ }^{1}, \mathrm{Ya} \mathrm{Cao}^{2}$, Jian Zhou ${ }^{1}$, Jia Fan ${ }^{1}$ and Shuang-Jian Qiü,1,3 \\ ${ }^{1}$ Liver Cancer Institute, Zhongshan Hospital and Shanghai Medical School, Fudan University, Key Laboratory for Carcinogenesis \\ and Cancer Invasion, The Chinese Ministry of Education, Shanghai 200032, People's Republic of China; ${ }^{2}$ Cancer Research Institute, \\ Xiangya School of Medicine, Central South University, Changsha, Hunan, People's Republic of China and ${ }^{3}$ Biomedical Research \\ Center, Zhongshan Hospital, Fudan University, 180 Fenglin Road, Shanghai 200032, People's Republic of China
}

Background: Aberrant expression of interleukin-35 (IL-35) has been implicated in dampening antitumour immunity. The aim of this study was to explore the prognostic significance of IL-35 expression in patients with hepatocellular carcinoma (HCC) following curative resection. Furthermore, we aimed to formulate an effective prognostic nomogram for HCC after hepatectomy.

Methods: Immunohistochemistry was applied to explore IL-35 expression as well as $\mathrm{CD}_{3} 9^{+} \mathrm{Foxp}^{+}$and Foxp3 ${ }^{+}$regulatory T cell (Treg) infiltration in tissue microarrays in primary cohort comprising 210 randomly selected HCC patients who underwent curative resection. The results were further verified in an independent validation cohort of $138 \mathrm{HCC}$ patients.

Results: Patients with higher expression of IL-35 are more likely to suffer postoperative recurrence. Interleukin-35 was also identified as an independent prognostic factor for recurrence free survival in multivariate analysis. No correlation was detected between IL-35 expression and Foxp3 ${ }^{+}$Treg infiltration, whereas significant positive correlation was found between IL-35 expression and $\mathrm{CD}_{39}{ }^{+} \mathrm{Foxp}^{+}$Treg infiltration. In addition, $\mathrm{CD}^{+} 9^{+} \mathrm{Foxp} 3^{+}$Treg infiltration was also an independent predictor for postoperative recurrence. The nomogram comprising tumour size, tumour vascular invasion, IL-35 and CD39 ${ }^{+}$Foxp3 ${ }^{+}$Tregs had better predictive accuracy when compared with BCLC stage for RFS. These results were further validated in the validation cohort.

Conclusions: Our data suggest for the first time that IL-35 expression correlates with HCC aggressiveness and emerged as a novel independent prognostic factor for recurrence, thus conferring the rationale to develop a novel therapy of targeting IL-35. Furthermore, IL-35 should be incorporated into nomogram to generate a more accurate predictive model.

Hepatocellular carcinoma (HCC) is the fifth most frequently diagnosed cancer, and the second leading cause of cancer-related deaths worldwide (Jemal et al, 2011). Although curative resection is applicable in selected candidates, $60-70 \%$ patients developed metastasis and recurrence within 5 years following surgery even with radical resection (Llovet et al, 2003). To make it worse, such high rate of recurrence and metastasis manifests a major challenge, and owing to that HCC is highly refractory to conventional chemotherapy and radiotherapy (Gish et al, 2007).

Intratumoral balance between CD8 ${ }^{+}$cytotoxic cells (CTLs) and regulatory T cells (Tregs) (Gao et al, 2007), impairment of tumourinfiltrating $\gamma \delta \mathrm{T}$ cells (Yi et al, 2013) as well as neutrophil infiltration in tumour ( $\mathrm{Li}$ et al, 2011) have been reported to be associated with survival in patients with HCC in our previous

\footnotetext{
*Correspondence: Professor S-J Qiu; E-mail: qiu.shuangjian@zs-hospital.sh.cn

${ }^{4}$ These authors contributed equally to this work.
}

Received 29 October 2015; revised 27 January 2016; accepted 9 February 2016; published online 22 March 2016

(c) 2016 Cancer Research UK. All rights reserved 0007-0920/16 
studies. These findings altogether implicate HCC as an immunogenic cancer and confer the rationale to develop immunotherapy as one of the alternative treatment strategies. Although immunedirected treatments such as cytokines, vaccines and activated immune cell infusions have been investigated for HCC treatment, only low clinical responses were reported (Makarova-Rusher et al, 2015). Accumulating evidence indicated that the immunosuppressive network within the tumour microenvironment is the major obstacle to the success of cancer immunotherapy (Hanahan and Weinberg, 2011). Cancer cells utilise multiple immunosuppressive mechanisms, predominately via the production of suppressive cytokines and induction of regulatory cells, to establish an immunosuppressive network within the tumour niche (Croci et al, 2007).

Interleukin-35 (IL-35) is a novel suppressive cytokine that is predominantly produced by Foxp ${ }^{+}$Tregs and is required for Treg-mediated immunosuppression (Collison et al, 2007). Interleukin-35 induces naive $\mathrm{T}$ cells to convert into Tregs as well as induces the conversion of human B cells into regulatory B cells (Bregs), and the converted cells play a key role in the negative regulation of immunity in autoimmune diseases (Collison et al, 2007, 2010; Wang et al, 2014). Interleukin-35 has also been complicated in tumour immunity as a novel regulatory cytokine that has potent effect on dampening $\mathrm{T}$ cell anticancer response (Collison et al, 2010). Recently, cancer cell-derived IL-35 was reported to promote tumour growth through the enhancement of myeloid cell accumulation and angiogenesis in tumour microenvironment (Wang et al, 2013b). High expression of IL-35 in tumour tissues and elevated plasma IL-35 levels indicated poor prognosis in several malignancies, including pancreatic ductal adenocarcinoma (Jin et al, 2014), colorectal cancer (Zeng et al, 2013), acute myeloid leukaemia (Wu et al, 2012) and non-smallcell lung cancer ( $\mathrm{Gu}$ et al, 2014). Intriguingly, a recent study has suggested that IL-35 exerts antitumour effect via inhibiting cancer cell growth and inducing apoptosis (Long et al, 2013). Therefore, further studies are needed to shed light on the specific immunological mechanisms that IL-35 mediated in diverse cancer cell types. Although expression of IL-35 in human cancers appears to be a fairly general phenomenon, the literature is currently devoid of clinical observations of IL-35 in human HCC.

The Tregs accumulating in multiple malignancies exert tumourpromoting effect via thwarting of antitumour immunity (Nishikawa and Sakaguchi, 2010). Previously, we showed that the intratumoral balance between CTLs and Tregs is associated with prognosis in HCC patients, and a balance toward Tregs was associated with poor outcome after resection (Gao et al, 2007). Foxp3 is currently the most reliable molecular marker for natural Tregs and provides clues with which to decipher the molecular and genetic basis of Treg development and function (Sakaguchi et al, 2008). Recently, a new Treg subset has been described based on its CD39 expression, an extracellular ectonucleoside triphosphate diphosphohydrolase-1 enzyme, capable of controlling activated lymphocytes through conversion of extracellular ATP (Borsellino et al, 2007), and CD39 was considered a promising therapeutic target for selectively inhibiting Treg function without depletion, a major limitation of current Treg-targeting strategies (Deaglio et al, 2007). More recently, an investigation suggested that CD39 ${ }^{+}$Tregs stimulated via IL-35 confer protection against collagen II-induced arthritis (Kochetkova et al, 2010). Thus, the relationship between IL-35 and CD39 ${ }^{+}$Tregs in HCC tissues is of great interest to be elucidated.

Nomograms are statistical models specifically developed to optimise predictive accuracy of individuals. Although other predictive models assign prognosis based on risk groups, nomograms provide a more individualised prediction of outcome based on a combination of variables. Currently, nomograms have been established in various cancer types (Bochner et al, 2006;
Sternberg, 2006). The predictive accuracy of nomograms has been considered superior to the traditional staging systems in many cancer populations, and thus it has been proposed as an alternative or even as a new standard (Wang et al, 2013a). However, nomograms in many cancer types were constructed mainly integrating clinicopathologic variables other than tumour biomarkers, further establishing that nomogram comprising IL-35/Tregs is significant.

To this end, it is logical to speculate that IL-35 expressed in HCC could contribute to poor prognosis. We also tried to uncover possible correlation between IL-35 expression and CD $39^{+}{ }^{+}$Foxp $3^{+}$Tregs. In addition, we aimed to develop a nomogram including IL-35/Tregs and compare its accuracy of prognostic prediction with BCLC stage in patients with HCC after curative resection.

\section{MATERIALS AND METHODS}

Patient selection and follow-up procedure. Two independent cohorts including a total of 348 patients with HCC who underwent curative resection in Zhongshan Hospital, Fudan University, in 2007 were enrolled and retrospectively analysed in this study. The patients were divided into two groups via digital random table: the first 210 were termed as training cohort and the remaining 138 as validation cohort. The inclusion and exclusion criteria for the patients analysed are as follows: (1) without any preoperative anticancer treatments that could introduce any bias; (2) exact diagnosis of pathologically proven HCC; (3) complete removal of the tumour without residual cancer defined as a complete resection of all tumour lesions and the cut surface being free of cancer by histological examination; (4) with complete clinicopathologic and follow-up data; (5) without any clinical evidence of infection or other inflammatory conditions other than viral hepatitis. All patients provided informed consent to participate in the study, and the study protocol was approved by the Clinical Research Ethic Committee of Zhongshan Hospital.

Conventional clinicopathologic variables comprising age, gender, hepatitis B virus surface antigen ( $\mathrm{HBsAg}$ ), liver cirrhosis, $\alpha$-fetoprotein (AFP), $\gamma$-glutamyl transferase (GGT), tumour size, number, vascular invasion, encapsulation, differentiation and BCLC stage were recorded and are detailed in Table 1. Postoperative surveillance and the treatment modality after relapse according to a uniform guideline were described in our previous study (Sun et al, 2007). Recurrence-free survival (RFS) time was defined as the interval between surgery and time of recurrence for relapsed patients or from the date of surgery to the date of last follow-up for nonrecurrent patients.

Tissue microarray and immunohistochemistry. Formalin-fixed, paraffin-embedded surgical specimens were histologically reviewed by HE staining and representative areas were selected precluding from necrotic and haemorrhagic tissue. Triplicate cores of $1 \mathrm{~mm}$ diameter were included for each surgical sample, and thus three different tissue microarray blocks were constructed and mounted on glass slides by sequencing.

Mouse anti-human IL-35 (Imgenex, Littleton, CO, USA), CD39 (Abcam, Cambridge, UK) and Foxp3 (Biolegend, San Diego, CA, USA) monoclonal antibodies were purchased. Immunohistochemistry (IHC) of serial tissue microarrays was performed as described previously (Gao et al, 2009).

Quantification of IL-35 expression and $\mathrm{CD}^{+}{ }^{+}{ }^{\text {Foxp }} 3^{+}$ immune cell infiltration. For IL-35, all samples were anonymised and independently scored by two investigators using the semiquantitative immunoreactivity scoring (IRS) system as described previously (Weichert et al, 2008). Immunoreactivity score was derived by multiplying the intensity of IHC staining, the percentage of immunoreactive cells ranged from 0 to 12 , and the 
Table 1. Correlation of intratumoral IL-35 expression and clinicopathologic characteristics in the training and validation set of patients with HCC

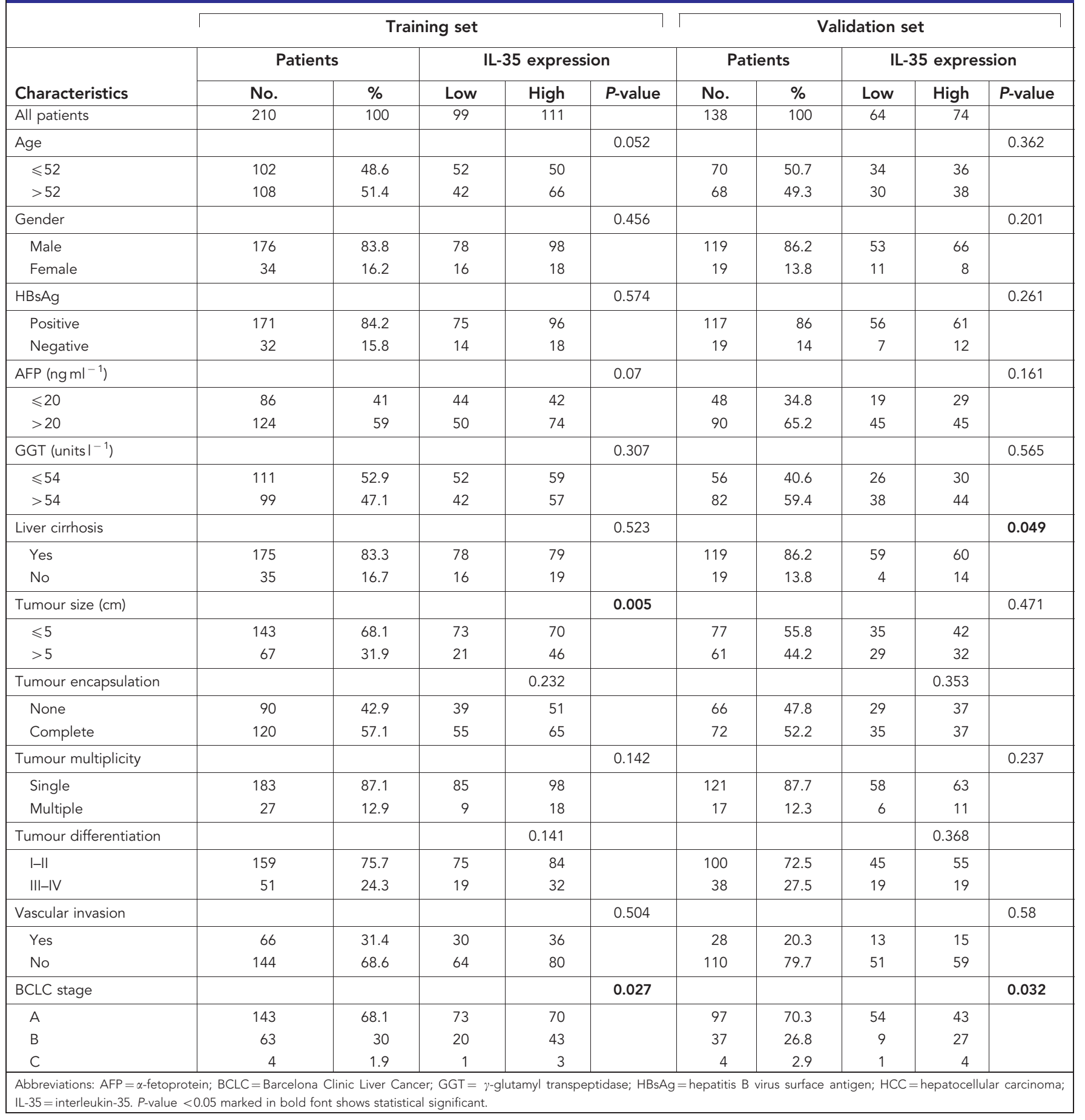

average score on each slide (three images) was used to represent a particular sample. As for $\mathrm{CD}_{3} 9^{+}$and Foxp $3^{+}$staining, the entire $1 \mathrm{~mm}$ core was counted manually and the average count per $1 \mathrm{~mm}$ disk was calculated. In case of disagreement, the slides were reviewed and a consensus was reached by the two observers. Finally, we defined 4 as the cutoff value of IL-35 and 2 of doublepositive $\mathrm{CD}_{39}{ }^{+} \mathrm{Foxp}^{+}$Tregs, respectively, for high and low expression according to the ' $\mathrm{x}$-tile analysis' based on their relation with RFS in training cohort, and then the cutoff values were further validated in an independent cohort.

Statistical analysis. Statistical analyses were performed with SPSS 19.0 (SPSS Inc., Chicago, IL, USA). The association between immunoreactive markers and clinicopathologic variables as well as the correlation between the density and $\mathrm{CD} 39^{+} \mathrm{Foxp}^{+}$tumourinfiltrating lymphocytes (TILs) was analysed using $\chi^{2}$ test or Fisher's exact test or $t$-test as appropriate. The survival curves were determined by the Kaplan-Meier analysis and compared by the log-rank test. The Cox proportional hazards regression model was used to perform univariate and multivariate analyses, and $P<0.05$ (two tailed) was considered statistically significant.

A nomogram was established based on the results of multivariate analysis and by using the package of rms in $\mathrm{R}$ version 2.14.1 (http://www.r-project.org/). The predictive accuracy of the nomogram was measured by concordance index (C-index) and assessed by comparing nomogram-predicted $v s$ observed Kaplan-Meier 
estimates of survival probability. The larger the C-index, the more accurate was the prognostic prediction (Huitzil-Melendez et al, 2010). Comparisons between the nomogram and BCLC stage as well as the external validation of nomogram was performed as previously described (Wang et al, 2013a).

\section{RESULTS}

Patient clinicopathologic profiles. The detailed characteristics of patients in training and validation cohorts are presented in Table 1, and the two independent sets were with an overall similar tumour burden. The median follow-up period was 53.4 months (range 1.5-61.3; s.d., 1.341). For the whole study population, RFS and overall survival (OS; in brackets) rates at 1, 3 and 5 years postoperative were $77.6 \%(89.7 \%), 61.2 \%(72.7 \%)$ and $48.9 \%$ (62.1\%). At last follow-up (28 February 2012), 178 (51.1\%) patients were diagnosed as relapse, and post-recurrent treatment modalities including reoperation $(n=35)$, transcatheter arterial chemoembolisation $(n=56)$, radiotherapy $(n=4)$, alternative Chinese traditional medicine $(n=4)$, radiofrequency ablation $(n=2)$ and percutaneous ethanol injection $(n=1)$ were administered as appropriate. However, 77 recurrent patients with severe liver dysfunction or weak general performance cannot stand any anticancer therapy.
IL-35 expression pattern in HCC tissue samples. To ascertain the manner of expression of IL-35 in HCC, tumour tissue and matched paratumour specimens of training and validation cohort were examined by IHC staining. In the tumour area, the expression of IL-35 was mainly localised in the cell cytoplasm, either in a focal or scattered pattern with variable staining intensity. For vast majority of HCC samples, IL-35 expression was evenly scattered throughout the specimens (Figures 1A-D), in concordance with their manner of expression in colorectal cancer (Zeng et al, 2013) and melanoma (Wang et al, 2013b). The expression level in tumour tissues was similar to paratumour tissues. In addition, IL35 expression was also identified in some infiltrating lymphocytes and endothelial cells in HCC tissues (Figures 1E and F).

Correlation between IL-35 expression and clinicopathologic features in HCC patients. To evaluate the correlation of IL-35 with tumour biology, comparisons of the clinicopathologic features with IL-35 expression were analysed in both training and validation cohorts. As shown in Table 1, high expression of IL35 was $52.9 \%$ (111 out of 210 ) and $53.6 \%$ (74 out of 138 ) in the training and validation sets, respectively. High IL-35 expression was associated with advanced BCLC stage $(P=0.006$ and 0.008 , respectively) in the two independent cohorts. In addition, in the training set, raised IL-35 expression was associated with larger tumour size $(P=0.005)$, and in validation set, high IL-35

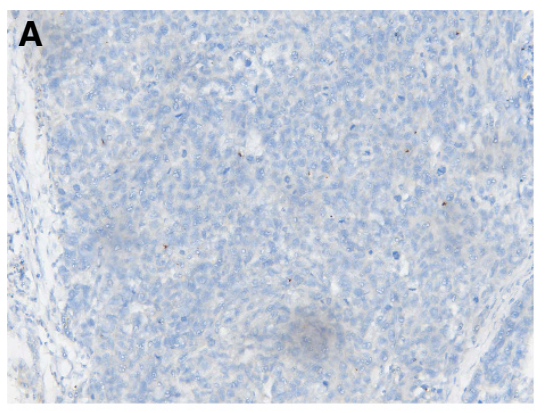

Intratumoral IL-35 negative

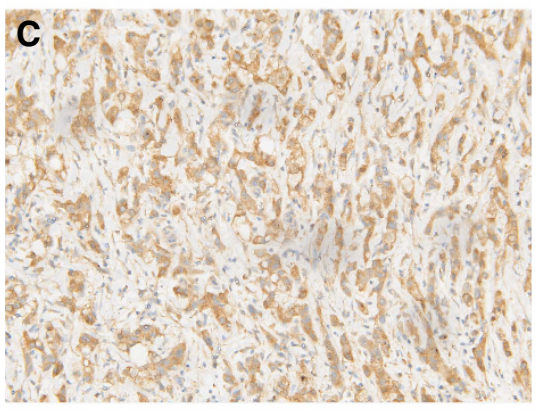

Intratumoral IL-35 moderate intensity

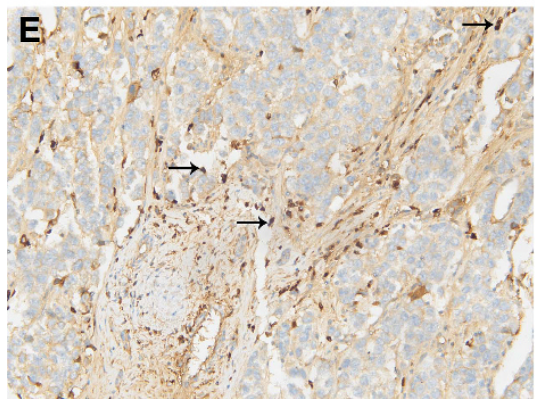

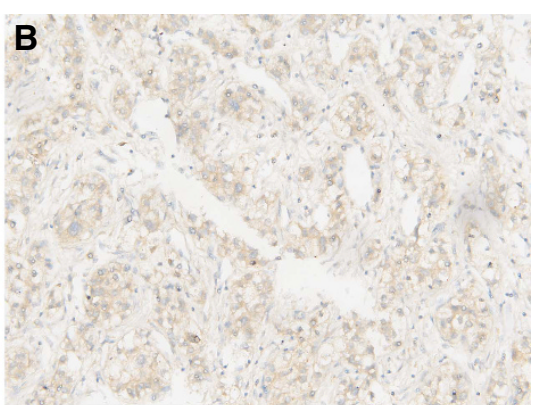

Intratumoral IL-35 weak intensity

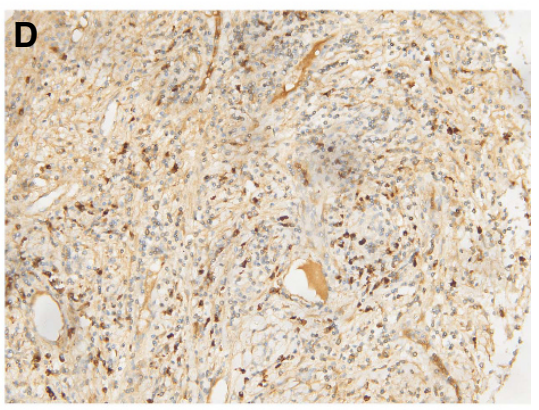

Intratumoral IL-35 strong intensity

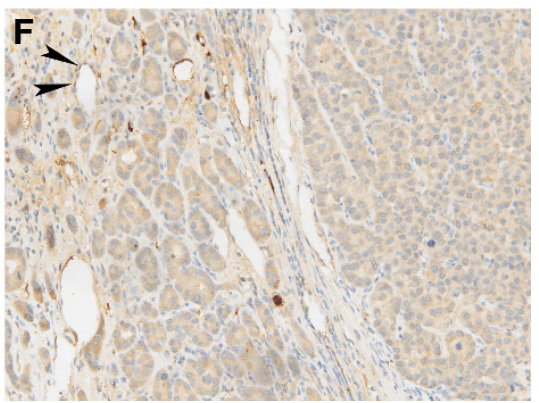

Figure 1. Interleukin-35 (IL-35) expression in sections of HCC tissue samples. In the tumour area, IL-35 expression was evenly scattered throughout the specimens. Representative microphotographs of IL-35 expression (A-F). Intratumoral negative control (A); intratumoral weak intensity (B); intratumoral moderate intensity (C); intratumoral strong intensity (D); tumour-infiltrating lymphocytes (arrow) (E); and endothelial cells (arrowhead) (F). Original magnification $\times 200$ 
Table 2. Univariate analyses of prognostic factors associated with recurrence-free survival

\begin{tabular}{|c|c|c|c|c|c|c|}
\hline \multirow[b]{3}{*}{ Variables } & \multicolumn{6}{|c|}{ Recurrence-free survival } \\
\hline & \multicolumn{3}{|c|}{ Training set } & \multicolumn{3}{|c|}{ Validation set } \\
\hline & HR & $95 \% \mathrm{Cl}$ & $P$-value & HR & $95 \% \mathrm{Cl}$ & $P$-value \\
\hline Age, years (>52 vs $\leqslant 52)$ & 1.275 & $0.867-1.875$ & 0.216 & 1.083 & $0.681-1.723$ & 0.735 \\
\hline Gender (female vs male) & 0.853 & $0.513-1.418$ & 0.547 & 0.947 & $0.496-1.808$ & 0.871 \\
\hline HBsAg (positive vs negative) & 2.43 & $1.226-4.819$ & 0.004 & 1.227 & $0.609-2.473$ & 0.557 \\
\hline AFP, $\mathrm{ng} \mathrm{ml}^{-1}(>20$ vs $\leqslant 20)$ & 1.755 & $1.162-2.650$ & 0.006 & 1.286 & $0.783-2.111$ & 0.314 \\
\hline GGT, units $\left.\right|^{-1}$ (>54 vs $\left.\leqslant 54\right)$ & 1.785 & $1.213-2.628$ & 0.003 & 1.735 & $1.056-2.849$ & 0.03 \\
\hline Liver cirrhosis (yes vs no) & 1.306 & $0.744-2.294$ & 0.337 & 1.002 & $0.478-1.729$ & 0.773 \\
\hline Tumour differentiation (III-IV vs I-II) & 1.756 & $1.161-2.655$ & 0.01 & 1.012 & $0.569-1.626$ & 0.885 \\
\hline Tumour size, $\mathrm{cm}(>5$ vs $\leqslant 5)$ & 2.318 & $1.566-3.431$ & $<0.001$ & 1.769 & $1.113-2.811$ & 0.016 \\
\hline Tumour multiplicity (multiple vs single) & 1.986 & $1.218-3.237$ & 0.01 & 1.134 & $0.581-2.217$ & 0.712 \\
\hline Tumour encapsulation (none vs complete) & 1.437 & $0.98-2.108$ & 0.065 & 1.001 & $0.54-1.361$ & 0.513 \\
\hline Vascular invasion (yes vs no) & 2.095 & $1.416-3.102$ & $<0.001$ & 2.058 & $1.225-3.457$ & 0.01 \\
\hline $\mathrm{BCLC}$ stage $(\mathrm{C}$ vs $\mathrm{B}$ vs $\mathrm{A})$ & 2.079 & $1.432-3.019$ & $<0.001$ & 2.215 & $1.447-3.391$ & 0.001 \\
\hline IL-35 (high vs low) & 2.764 & $1.796-4.252$ & $<0.001$ & 2.718 & $1.630-4.535$ & $<0.001$ \\
\hline Foxp $3^{+}$TILs (high vs low) & 1.715 & $1.026-2.865$ & 0.03 & 1.755 & $0.868-3.550$ & 0.094 \\
\hline CD39 $^{+}$Foxp $^{+}{ }^{+}$TILs (high vs low) & 1.842 & $1.134-2.991$ & 0.009 & 2.077 & $1.027-4.201$ & 0.027 \\
\hline
\end{tabular}

Abbreviations: $\mathrm{AFP}=\alpha$-fetoprotein; $\mathrm{BCLC}=$ Barcelona Clinic Liver Cancer; $\mathrm{Cl}=$ confidence interval; $\mathrm{GGT}=\gamma$-glutamyl transpeptidase; $\mathrm{HBsAg}=$ hepatitis $\mathrm{B}$ virus surface antigen; $\mathrm{HR}=$ hazard ratio; IL-35= interleukin-35; TIL = tumour-infiltrating lymphocyte. $P$-value $<0.05$ marked in bold font shows statistical significant.

expression was correlated with absence of liver cirrhosis $(P=0.049)$

The significance of IL-35 expression and CD39 ${ }^{+}$Foxp3 $^{+}$Tregs in RFS of patients with HCC. High intratumoral IL-35 expression patients were revealed to have significantly poorer RFS and OS than low IL-35 expression patients in the two independent cohorts (Table 2 and Figures $2 \mathrm{~A}$ and $\mathrm{D}$ ). In multivariate analysis, tumour IL-35 status was found to be an independent prognostic factor for RFS. The IL-35-high patients were nearly 3 times more likely to suffer from recurrence than IL35-low patients in the two independent sets (hazard ratio (HR), 2.878; $95 \%$ confidence interval (95\% CI), 1.925-4.929 and HR, 2.874; 95\% CI, 1.725-5.196; respectively, Table 3). However, the significant differences in OS disappeared on multivariate analysis.

To further investigate the significance of IL-35 expression in discriminating patients with different clinicopathologic features, we performed subgroup analysis between IL-35-high and IL-35low patients categorised by tumour size, number, vascular invasion, differentiation, encapsulation and AFP level in the two independent cohorts. Interleukin-35 was identified to stratify patient recurrence regarding small tumour, large tumour, single tumour, well-differentiated tumour, tumour with/without encapsulation, tumour without vascular invasion and normal/elevated AFP level in both training and validation cohorts (Supplementary Table S1 and Supplementary Figures S1 and S2).

We also investigated the effect of $\mathrm{CD}_{3}{ }^{+}{ }^{+}$Foxp $3{ }^{+}$Tregs in stratifying patient outcome in terms of RFS in the two independent cohorts. Representative images of $\mathrm{CD}_{3} 9^{+}$Foxp $3^{+}$Tregs are shown in Supplementary Figure S3. In univariate analysis, significant differences in recurrence were found between high-dose CD39 ${ }^{+}$ Foxp $^{+}$Tregs and low-dose CD $39^{+}$Foxp $3^{+}$Tregs (Table 2 and Figures $2 \mathrm{~B}$ and E). In multivariate analysis, CD $39^{+}$Foxp $3^{+}$Treg count was found to be an independent prognostic factor for RFS. The CD $39^{+}{ }^{\text {Foxp }}{ }^{+}$Treg-high patients were nearly 2 times more likely to suffer from recurrence than $\mathrm{CD} 39^{+}$Foxp3 ${ }^{+}$Treg-low patients in the two independent sets (HR, 1.664; 95\% CI, 1.012 2.737 and HR, 1.94; 95\%CI, 0.954-3.944; respectively, Table 3). In univariate analysis, significant differences in OS was found between high-dose $\mathrm{CD} 39^{+}{ }^{+}$Foxp $3^{+}$Tregs and low-dose CD $39^{+}$ Foxp $3^{+}$Tregs, but the significant differences in OS disappeared on multivariate analysis.

Furthermore, we investigated the significance of combined IL35 expression and $\mathrm{CD} 39^{+}$Foxp $3^{+}$Tregs in patient prognosis. Patients were divided into three groups: (A) both IL-35 and $\mathrm{CD}{ }^{+}$Foxp $3^{+}$Tregs were high; (B) either IL-35 or $\mathrm{CD} 39^{+}$ Foxp $3^{+}$Tregs was high; (C) both IL- 35 and CD $39^{+}$Foxp ${ }^{+}$Tregs were low. Significant differences in recurrence was found between groups $\mathrm{A}$ and $\mathrm{B}$ as well as between groups $\mathrm{B}$ and $\mathrm{C}(P=0.028$ and $P<0.001$, respectively; Figures $2 \mathrm{C}$ and $\mathrm{F}$ ); intriguingly, more remarkably disparity was observed between groups $\mathrm{A}$ and C compared with the difference between IL-35-high and IL-35-low patients or between $\mathrm{CD}_{3}{ }^{+}$Foxp $^{+}$Treg-high and CD39 ${ }^{+}$ Foxp $^{+}$Treg-low groups in both training and validation sets (Figures 2C and F).

Correlation between IL-35 expression and Tregs. The count of Foxp $3^{+}$Tregs was found to differentiate recurrence in univariate analysis, but the significance in discriminating prognosis failed in multivariate analysis, whereas the count of double-positive of CD $39^{+}$Foxp $3^{+}$Tregs was defined as an independent prognostic factor for RFS in multivariate analysis and further validated in an independent cohort (Tables 2 and 3 and Figures 2B and E). Then we tried to uncover the possible correlation between IL-35 and Foxp $3^{+} / \mathrm{CD} 39^{+}$Foxp $3^{+}$Tregs. There was no significant correlation between IL-35 and Foxp $3^{+}$Tregs $(P=0.125)$. Intriguingly, significant positive correlation between IL-35 expression and $\mathrm{CD} 9^{+}{ }^{+}$Foxp ${ }^{+}$Tregs was found in primary cohort $(P=0.044)$ and further verified in validation cohort $(P=0.046)$.

Prognostic nomogram for RFS. To generate a more accurate predictive model, we integrated the independent prognostic factors in both primary and validation cohorts (Tables 2 and 3 ) to create a prognostic nomogram (Figure $3 \mathrm{~A}$ ). The C-index for RFS prediction of the formulated nomogram in primary cohort is $0.7085(95 \%$ CI, 0.7017-0.715) and the points assigned to each variable value are shown in Supplementary Table S2. The calibration plot for the 

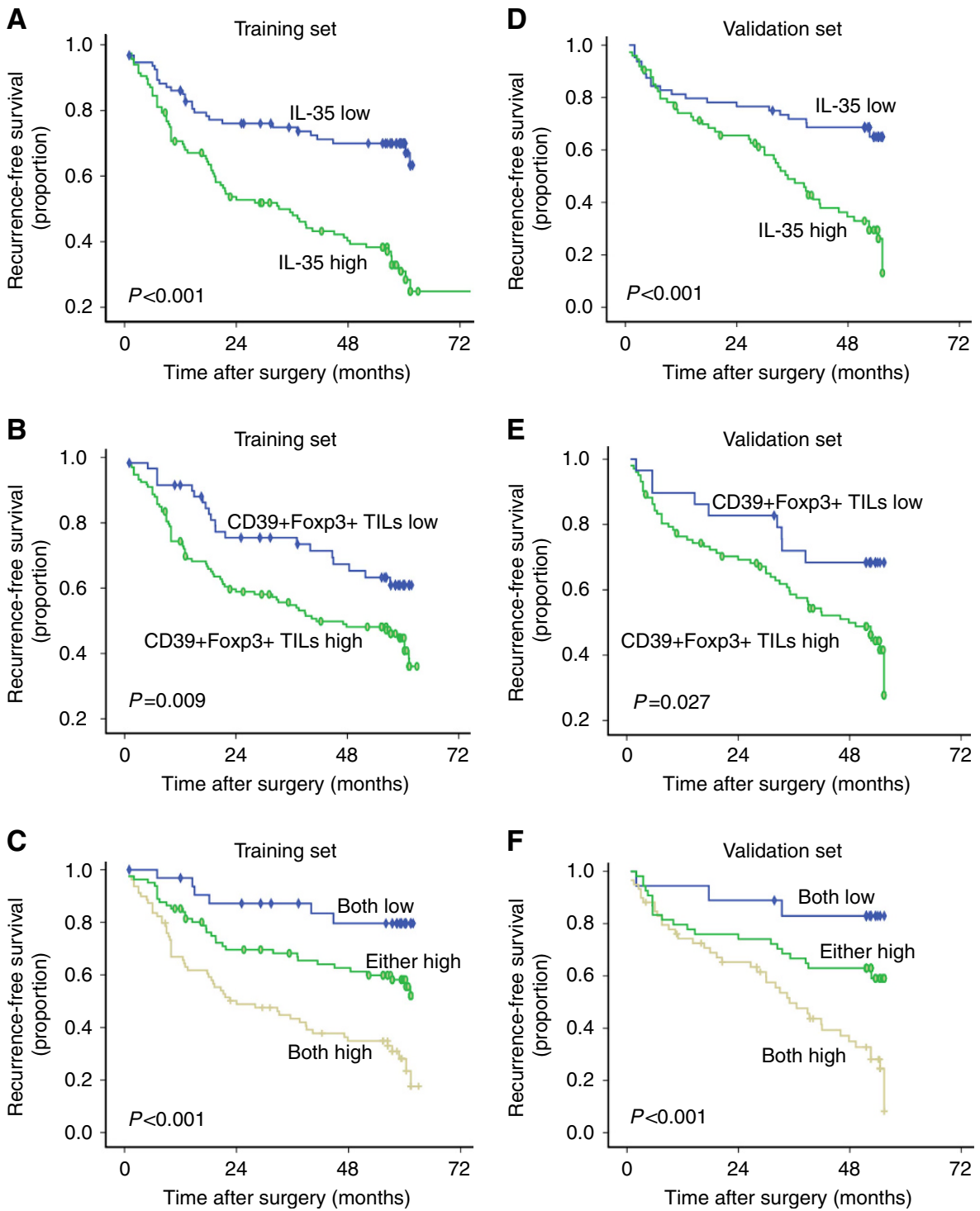

Figure 2. Kaplan-Meier curves for RFS of patients with HCC according to the expression of IL-35 and the number of infiltrating CD39 ${ }^{+}$Foxp $3^{+}$ Tregs. Recurrence-free survival (RFS) for expression of IL-35 (A), the number of infiltrating CD39 ${ }^{+}$Foxp3 ${ }^{+}$Tregs (B) and their combination (C) was found to be statistically significant. Significant differences in RFS were further validated in an independent validation cohort (D-F). The $P$-values were determined by the log-rank test.

\section{Table 3. Multivariate analyses of prognostic factors associated with recurrence-free survival}

\begin{tabular}{|c|c|c|c|c|c|c|}
\hline \multirow[b]{3}{*}{ Variables } & \multicolumn{6}{|c|}{ Recurrence-free survival } \\
\hline & \multicolumn{3}{|c|}{ Training set } & \multicolumn{3}{|c|}{ Validation set } \\
\hline & HR & $95 \% \mathrm{Cl}$ & $P$-value & HR & $95 \% \mathrm{Cl}$ & $P$-value \\
\hline AFP, $\mathrm{ng} \mathrm{ml}^{-1}(>20$ vs $\leqslant 20)$ & 1.471 & $0.939-2.304$ & 0.092 & & & \\
\hline GGT, units $I^{-1}(>54$ vs $\leqslant 54)$ & 1.522 & $0.987-2.346$ & 0.057 & 1.372 & $0.787-2.390$ & 0.265 \\
\hline Tumour multiplicity (multiple vs single) & 1.603 & $0.939-2.738$ & 0.084 & & & \\
\hline Vascular invasion (yes vs no) & 1.798 & $1.162-2.783$ & 0.008 & 2.044 & $1.183-3.531$ & 0.002 \\
\hline IL-35 (high vs low) & 2.878 & $1.925-4.929$ & 0.002 & 2.874 & $1.725-5.196$ & 0.003 \\
\hline Foxp3 ${ }^{+}$TILs (high vs low) & 1.357 & $0.788-2.337$ & 0.271 & & & \\
\hline $\mathrm{CD}^{2} 9^{+}$Foxp3 $^{+}$TILs (high vs low) & 1.664 & $1.012-2.737$ & 0.045 & 1.94 & $0.954-3.944$ & 0.046 \\
\hline
\end{tabular}


A
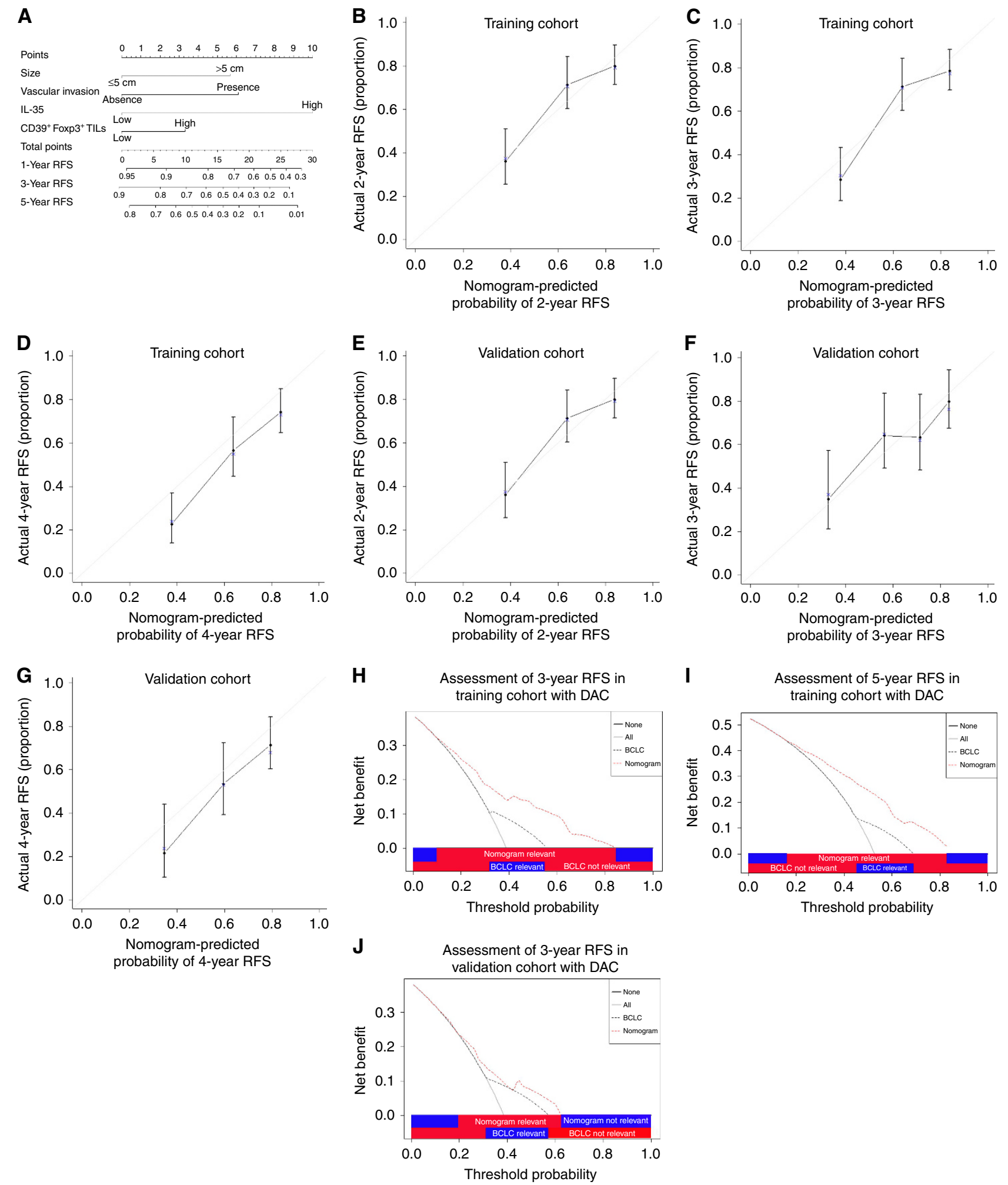

Figure 3. Hepatocellular carcinoma (HCC) RFS nomogram, calibration curve and decision curve analysis. (A) Hepatocellular carcinoma (HCC) RFS nomogram (to use the nomogram, an individual patient's value is located on each variable axis, and a line is drawn upwards to determine the number of points received for each variable value. The sum of these numbers is located on the Total Points axis, and a line is drawn downwards to the survival axes to determine the likelihood of 3 - or 5 -year RFS). The calibration curve for predicting RFS at (B) 3 years, (C) 4 years and (D) 5 years in training cohort and at (E) 3 years, $(\mathbf{F}) 4$ years and $(\mathbf{G}) 5$ years in the validation cohort. Nomogram-predicted probability of overall survival is plotted on the $\mathrm{x}$ axis and actual overall survival is plotted on the $\mathrm{y}$ axis. Decision curve analyses depict the clinical net benefit in pairwise comparisons across the different models. Nomogram is compared with the BCLC stage in terms of (H) 3-year and (I) 5-year RFS in training cohort and (J) 5-year RFS in validation cohort. Dashed lines indicate the net benefit of nomogram in each of the curves across a range of threshold probabilities. The horizontal solid black line represents the assumptions that no patient will experience the event, and the solid grey line represents the assumption that all patients will relapse. On decision curve analysis, nomogram showed superior net benefit compared with BCLC stage across a range of threshold probabilities. 
probability of RFS at 3,4 or 5 years after surgery showed optimal consistency between the prediction by nomogram and actual observation (Figures 3B-D).

The predictive accuracy of the nomogram for RFS was further verified in the validation cohort. The $\mathrm{C}$-index of the constructed nomogram derived from primary cohort for RFS prediction in the validation cohort is 0.6735 (95\% CI, 0.661-0.686), and the calibration curve fits well between prediction and observation in the probability of 3, 4 and 5-year RFS (Figures 3E-G).

Given that the nomogram comprising tumour size, vascular invasion, $\mathrm{CD} 39^{+}{ }^{-}$Foxp $3^{+}$Tregs and IL-35 expression demonstrated superior predictive capabilities relative to the BCLC stage, with a C-index of 0.7085 (95\% CI, 0.7017-0.715) and 0.581 (95\% CI, 0.574-0.588) respectively for prediction of RFS, comparison of constructed nomogram with BCLC stage as predictor of RFS on decision curve analysis (Vickers and Elkin, 2006) was necessary to be performed. On decision curve analysis, compared with BCLC stage, nomogram showed better net benefit with wider range of threshold probability and improved performance for predicting 3- and 5-year RFS in training and 5-year RFS in validation cohort, respectively. This further represents superior estimation of decision outcomes at higher threshold probability levels (Figures $3 \mathrm{H}-\mathrm{J}$ ).

\section{DISCUSSION}

Herein, we demonstrated that IL-35, an immunosuppressive cytokine, is constitutively expressed in HCC specimens. More importantly, we performed the first large-scale study via highthroughput tissue microarray analysis to evaluate the prognostic capacity of tumour IL-35 expression in randomly selected population of patients with HCC after curative resection and further in an independent validation cohort. Furthermore, a novel nomogram comprising independent prognostic factors including tumour size, vascular invasion, IL- 35 and $\mathrm{CD} 39^{+}{ }^{+}$Foxp $3{ }^{+}$Tregs in both data sets was constructed and showed improved prognostic accuracy relative to traditional BCLC stage for RFS in terms of C-index.

First, we validated the hypothesis that IL-35 is produced in HCC tissues. Interleukin-35 was found expressed in HCC tumour cells as well as tumour stromal cells, in concordance with previous findings that IL-35 can be derived from multiple cell types including diffuse large B lymphoma cells (Wang et al, 2013b), colorectal cancer cells (Zeng et al, 2013) and tumour-infiltrating Foxp $3^{+}$lymphocytes (Collison et al, 2007).

Next, we verified the speculation that IL-35 expressed in the tumour microenvironment may contribute to poor prognosis. We found that high IL-35 expression was more likely observed in patients with advanced BCLC stage and at significant high risk of cancer recurrence. In multivariate analysis, IL-35 expression was strengthened as an independent prognostic factor along with the well-established variables including tumour size, vascular invasion and BCLC stage and was further verified in an independent cohort of patients with similar follow-up period and tumour burden. This result was consistent with the previous findings in other cancer types (Zeng et al, 2013; Gu et al, 2014; Jin et al, 2014) and implied the potential role of IL-35 in tumour progression. The basis for the results may relate to the recognised ability of IL-35 to sustain the suppressive function of multiple immunosuppressive cells in vitro and in vivo. In vivo, IL-35 secreted by Tregs can also induce naive $\mathrm{T}$ cells to convert into Tregs (iTr35 cells) (Collison et al, 2010). Thus, IL-35 mediates the biology function of Tregs and triggers the genesis of more Tregs that maximise the suppressive activity. Furthermore, a recent study found that IL-35 production in the tumour environment increased myeloid-derived suppressor cell accumulation (Wang et al, 2013b) that induce immune suppression and inhibit CTL responses (Nagaraj and Gabrilovich, 2008; Gabrilovich and Nagaraj, 2009). Taken together, IL-35 is an important factor that facilitates establishment of suppressive network that may favour the balance between CTL and Tregs towards Tregs, and this would lead to recurrence as evidenced by our previous study (Gao et al, 2007).

We also examined the correlation between IL-35 expression and number of Tregs, and then consequently the significance of Tregs alone or combined with IL-35 in prediction of HCC outcome. Firstly, no significant correlation was observed between IL-35 expression and the number of Foxp $3^{+}$Tregs; the underlying mechanism may be that Foxp3 is currently the most reliable molecular marker for natural but not induced Tregs (Sakaguchi et al, 2008). As an investigation suggested that IL-35 stimulation of $\mathrm{CD}^{2}{ }^{+}$Tregs confers protection against collagen II-induced arthritis (Kochetkova et al, 2010), accumulating evidence indicated that CD39 expressed on Tregs mediates immune suppression in autoimmune disease (Borsellino et al, 2007; Sauer et al, 2012) as well as within tumour microenvironment (Bastid et al, 2013), and more recently CD39 + Foxp3 + Tregs were found to be a source of IL-35 (Kochetkova et al, 2014). Taken together, it will be interesting to determine the correlation between IL-35 expression and the number of $\mathrm{CD} 39^{+}$Foxp $3^{+}$Tregs. Notably, significant positive correlation between IL-35 expression and CD39 ${ }^{+}$Foxp $3^{+}$ Tregs was found and further validated. This suggests that IL-35induced tumour immune suppression may operate through the arm of $\mathrm{CD}_{3} 9^{+} \mathrm{Foxp}^{+}$Tregs, although their causal link and precise nature warrant further investigation. Secondly, although Foxp $^{+}$Tregs correlated with tumour recurrence, it was not significant anymore on multivariate analysis, conferring a validation of our previous study (Gao et al, 2007). Intriguingly, CD39 ${ }^{+}$ $\mathrm{Foxp}^{+}$Treg was identified as independent prognostic factor for RFS prediction; more importantly, when combined with IL-35, more remarkable disparity between different groups was observed.

In line with the predictive accuracy and superiority of nomograms as compared with the traditional staging systems in other cancer populations (Bochner et al, 2006; Wang et al, 2013a), we found that our constructed nomogram including IL-35 and Tregs had better predictive accuracy when compared with the BCLC stage. The result is provocative in suggesting that immune status within neoplastic niche should be incorporated into HCC prognostic system to improve the discriminative ability.

Several shortcomings should be considered as follows. Firstly, the study is retrospective in nature and all the data were collected from a single institution in China. Secondly, whether IL-35 can be applied to patients who receive treatment other than curative resection remains to be determined. Nonetheless, more prospective studies should be conducted to further validate the predicting probability of IL-35 and this nomogram.

In conclusion, despite the acknowledged limitations, these data suggest that IL-35 correlates with HCC aggressiveness and can be applied as a novel prognostic factor for RFS of patients. Furthermore, the proposed nomogram including IL-35 and CD39+ $\mathrm{Foxp}^{+}$Tregs presents a better prognostic model. 'As such, IL-35 may represent a target for HCC immunotherapy and a potential biomarker to facilitate patients in the prediction of recurrence.

\section{ACKNOWLEDGEMENTS}

This work was in part supported by the National Key Sci-Tech Special Project of China (Grant No. 2012ZX10002010-001/002); the National Natural Science Foundation of China (Grant No. 81302102); and the Basic Research Programs of Science and 
Technology Commission Foundation of Shanghai (Grant No. 13JC1401800, XBR2013074).

\section{CONFLICT OF INTEREST}

The authors declare no conflict of interest.

\section{AUTHOR CONTRIBUTIONS}

Conceived and designed the experiments: Shuang-Jian Qiu, Jian Zhou and Jia Fan. Performed the experiments: Yi-Peng Fu, Yong Yi and Xiao-Yan Cai. Statistically analysed the data: Yi-Peng $\mathrm{Fu}$ and Yong Yi. Contributed reagents/materials/analysis tools: Jian Sun, Xiao-Chun Ni, Hong-Wei He, Jia-Xing Wang, Ya Cao, JinLong Huang and Zhu-Feng Lu. Wrote the paper: Yi-Peng Fu and Yong Yi.

\section{REFERENCES}

Bastid J, Cottalorda-Regairaz A, Alberici G, Bonnefoy N, Eliaou JF Bensussan A (2013) ENTPD1/CD39 is a promising therapeutic target in oncology. Oncogene 32: 1743-1751.

Bochner BH, Kattan MW, Vora KC (2006) Postoperative nomogram predicting risk of recurrence after radical cystectomy for bladder cancer. J Clin Oncol 24: 3967-3972.

Borsellino G, Kleinewietfeld M, Di Mitri D, Sternjak A, Diamantini A, Giometto R, Hopner S, Centonze D, Bernardi G, Dell'Acqua ML, Rossini PM, Battistini L, Rotzschke O, Falk K (2007) Expression of ectonucleotidase CD39 by Foxp3 + Treg cells: hydrolysis of extracellular ATP and immune suppression. Blood 110: 1225-1232.

Collison LW, Chaturvedi V, Henderson AL, Giacomin PR, Guy C, Bankoti J, Finkelstein D, Forbes K, Workman CJ, Brown SA, Rehg JE, Jones ML, Ni HT, Artis D, Turk MJ, Vignali DA (2010) IL-35-mediated induction of a potent regulatory T cell population. Nat Immunol 11: 1093-1101.

Collison LW, Workman CJ, Kuo TT, Boyd K, Wang Y, Vignali KM, Cross R, Sehy D, Blumberg RS, Vignali DA (2007) The inhibitory cytokine IL-35 contributes to regulatory T-cell function. Nature 450: 566-569.

Croci DO, Zacarias Fluck MF, Rico MJ, Matar P, Rabinovich GA, Scharovsky OG (2007) Dynamic cross-talk between tumor and immune cells in orchestrating the immunosuppressive network at the tumor microenvironment. Cancer Immunol Immunother 56: 1687-1700.

Deaglio S, Dwyer KM, Gao W, Friedman D, Usheva A, Erat A, Chen JF, Enjyoji K, Linden J, Oukka M, Kuchroo VK, Strom TB, Robson SC (2007) Adenosine generation catalyzed by CD39 and CD73 expressed on regulatory T cells mediates immune suppression. J Exp Med 204: $1257-1265$.

Gabrilovich DI, Nagaraj S (2009) Myeloid-derived suppressor cells as regulators of the immune system. Nat Rev Immunol 9: 162-174.

Gao Q, Qiu SJ, Fan J, Zhou J, Wang XY, Xiao YS, Xu Y, Li YW, Tang ZY (2007) Intratumoral balance of regulatory and cytotoxic T cells is associated with prognosis of hepatocellular carcinoma after resection. J Clin Oncol 25: 2586-2593.

Gao Q, Wang XY, Qiu SJ, Yamato I, Sho M, Nakajima Y, Zhou J, Li BZ, Shi YH, Xiao YS, Xu Y, Fan J (2009) Overexpression of PD-L1 significantly associates with tumor aggressiveness and postoperative recurrence in human hepatocellular carcinoma. Clin Cancer Res 15: 971-979.

Gish RG, Porta C, Lazar L, Ruff P, Feld R, Croitoru A, Feun L, Jeziorski K, Leighton J, Gallo J, Kennealey GT (2007) Phase III randomized controlled trial comparing the survival of patients with unresectable hepatocellular carcinoma treated with nolatrexed or doxorubicin. J Clin Oncol 25: 3069-3075.

Gu X, Tian T, Zhang B, Liu Y, Yuan C, Shao L, Guo Y, Fan K (2014) Elevated plasma interleukin-35 levels predict poor prognosis in patients with non-small cell lung cancer. Tumour Biol 36(4): 2651-2656.

Hanahan D, Weinberg RA (2011) Hallmarks of cancer: the next generation. Cell 144: 646-674.
Huitzil-Melendez FD, Capanu M, O’Reilly EM, Duffy A, Gansukh B, Saltz LL, Abou-Alfa GK (2010) Advanced hepatocellular carcinoma: which staging systems best predict prognosis? J Clin Oncol 28: 2889-2895.

Jemal A, Bray F, Center MM, Ferlay J, Ward E, Forman D (2011) Global cancer statistics. CA Cancer J Clin 61: 69-90.

Jin P, Ren H, Sun W, Xin W, Zhang H, Hao J (2014) Circulating IL-35 in pancreatic ductal adenocarcinoma patients. Hum Immunol 75: 29-33.

Kochetkova I, Golden S, Holderness K, Callis G, Pascual DW (2010) IL-35 stimulation of CD39 + regulatory $\mathrm{T}$ cells confers protection against collagen II-induced arthritis via the production of IL-10. J Immunol 184: 7144-7153.

Kochetkova I, Thornburg T, Callis G, Holderness K, Maddaloni M, Pascual DW (2014) Oral Escherichia coli colonization factor antigen I fimbriae ameliorate arthritis via IL-35, not IL-27. J Immunol 192: 804-816.

Li YW, Qiu SJ, Fan J, Zhou J, Gao Q, Xiao YS, Xu YF (2011) Intratumoral neutrophils: a poor prognostic factor for hepatocellular carcinoma following resection. J Hepatol 54: 497-505.

Llovet JM, Burroughs A, Bruix J (2003) Hepatocellular carcinoma. Lancet 362 : 1907-1917.

Long J, Zhang X, Wen M, Kong Q, Lv Z, An Y, Wei XQ (2013) IL-35 over-expression increases apoptosis sensitivity and suppresses cell growth in human cancer cells. Biochem Biophys Res Commun 430: 364-369.

Makarova-Rusher OV, Medina-Echeverz J, Duffy AG, Greten TF (2015) The yin and yang of evasion and immune activation in HCC. J Hepatol 62: $1420-1429$.

Nagaraj S, Gabrilovich DI (2008) Tumor escape mechanism governed by myeloid-derived suppressor cells. Cancer Res 68: 2561-2563.

Nishikawa H, Sakaguchi S (2010) Regulatory T cells in tumor immunity. Int J Cancer 127: 759-767.

Sakaguchi S, Yamaguchi T, Nomura T, Ono M (2008) Regulatory T cells and immune tolerance. Cell 133: 775-787.

Sauer AV, Brigida I, Carriglio N, Hernandez RJ, Scaramuzza S, Clavenna D, Sanvito F, Poliani PL, Gagliani N, Carlucci F, Tabucchi A, Roncarolo MG, Traggiai E, Villa A, Aiuti A (2012) Alterations in the adenosine metabolism and CD39/CD73 adenosinergic machinery cause loss of Treg cell function and autoimmunity in ADA-deficient SCID. Blood 119: 1428-1439.

Sternberg CN (2006) Are nomograms better than currently available stage groupings for bladder cancer? J Clin Oncol 24: 3819-3820.

Sun HC, Zhang W, Qin LX, Zhang BH, Ye QH, Wang L, Ren N, Zhuang PY, Zhu XD, Fan J, Tang ZY (2007) Positive serum hepatitis B e antigen is associated with higher risk of early recurrence and poorer survival in patients after curative resection of hepatitis B-related hepatocellular carcinoma. J Hepatol 47: 684-690.

Vickers AJ, Elkin EB (2006) Decision curve analysis: a novel method for evaluating prediction models. Med Decis Making 26: 565-574.

Wang RX, Yu CR, Dambuza IM, Mahdi RM, Dolinska MB, Sergeev YV, Wingfield PT, Kim SH, Egwuagu CE (2014) Interleukin-35 induces regulatory B cells that suppress autoimmune disease. Nat Med 20: 633-641.

Wang Y, Li J, Xia Y, Gong R, Wang K, Yan Z, Wan X, Liu G, Wu D, Shi L, Lau W, Wu M, Shen F (2013a) Prognostic nomogram for intrahepatic cholangiocarcinoma after partial hepatectomy. J Clin Oncol 31: 1188-1195.

Wang Z, Liu JQ, Liu Z, Shen R, Zhang G, Xu J, Basu S, Feng Y, Bai XF (2013b) Tumor-derived IL-35 promotes tumor growth by enhancing myeloid cell accumulation and angiogenesis. J Immunol 190 2415-2423.

Weichert W, Roske A, Gekeler V, Beckers T, Ebert MP, Pross M, Dietel M, Denkert C, Rocken C (2008) Association of patterns of class I histone deacetylase expression with patient prognosis in gastric cancer: a retrospective analysis. Lancet Oncol 9: 139-148.

Wu H, Li P, Shao N, Ma J, Ji M, Sun X, Ma D, Ji C (2012) Aberrant expression of Treg-associated cytokine IL-35 along with IL-10 and TGF-beta in acute myeloid leukemia. Oncol Lett 3: 1119-1123.

Yi Y, He HW, Wang JX, Cai XY, Li YW, Zhou J, Cheng YF, Jin JJ, Fan J, Qiu SJ (2013) The functional impairment of HCC-infiltrating gammadelta $\mathrm{T}$ cells, partially mediated by regulatory $\mathrm{T}$ cells in a TGFbeta- and IL-10-dependent manner. J Hepatol 58: 977-983. 
Zeng JC, Zhang Z, Li TY, Liang YF, Wang HM, Bao JJ, Zhang JA, Wang WD, Xiang WY, Kong B, Wang ZY, Wu BH, Chen XD, He L, Zhang S,

Wang CY, Xu JF (2013) Assessing the role of IL-35 in colorectal cancer progression and prognosis. Int J Clin Exp Pathol 6: 1806-1816.
This work is published under the standard license to publish agreement. After 12 months the work will become freely available and the license terms will switch to a Creative Commons AttributionNonCommercial-Share Alike 4.0 Unported License.

Supplementary Information accompanies this paper on British Journal of Cancer website (http://www.nature.com/bjc) 